\title{
ÜBER DEN QUASINORMALTEILER DER REGULÄREN $p$-GRUPPE VON DER KLASSE 2
}

\author{
KIRIO NAKAMURA
}

In der vorliegenden Arbeit wird untersucht, was für eine Struktur eine Untergruppe $\mathfrak{N}$ von endlicher $p$-Gruppe $\mathbb{S}$ habe, wenn $\mathfrak{N}$ mit jeder Untergruppe von $\mathbb{B}$ als ganzes vertauschbar ist. In diesen $\mathfrak{N}$ tritt natürlich ein nicht nur aus $E$ bestehender Normalteiler von $\&$ auf und er enthält ein Zentrumselement von der Ordnung $p$. Wir fragen: Gilt das Ähnliche auch, falls $\Re$ kein Normalteiler ist? Die gestellte Frage läßt sich für regülare $\$$ beantworten, deren Klasse 2 ist, aber für andere bleibt sie noch offen. Bevor wir diese Frage untersuchen, stellen wir einige Definitionen und nötige Sätze und Hilfssätze zusammen.

\section{Bezeichnungen}

Im folgenden bedeuten $\mathfrak{A}, \mathfrak{B}, \ldots$ eine Gruppe von endlicher Ordnung $|\mathfrak{A}|,|\mathfrak{B}|, \ldots$ und $A, B, \ldots$ ihre Elemente mit der Ordnung $|A|,|B|, \ldots$ Das Erzeugnis von $A, B, \ldots$ bezeichnen wir mit $\{A, B, \ldots\}$. Das Zentrum oder die Kommutatorgruppe von $\mathbb{B}$ bezeichnen wir mit $B$ oder $(\mathcal{B}$ '. Ist $\mathfrak{A}$ und $\mathfrak{B}$ als ganzes vertauschbar, so bezeichnen wir mit $\mathscr{U} \mathfrak{B} B$ und das Produkt $\mathscr{A} \mathfrak{B}$ ist bekanntlich eine Gruppe. Ist $\mathfrak{N} \mathscr{\mathscr { S }}=\mathscr{S} \mathfrak{N}$ für ein festes $\mathfrak{R}$ und für ein beliebiges $\mathscr{S} \subseteq \mathbb{B}$, so heißt $\Re$ quasi-normal in $\mathbb{B}$ oder ein Quasinormalteiler von $\mathbb{B}$ und

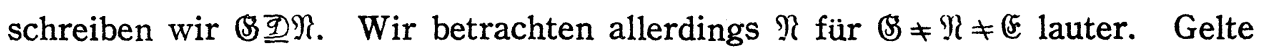
ebenso für ein festes $N \in \&$ mit $|N|=p$ und ein beliebiges $G \in B[N, G]=G^{p^{g-1}}$ oder $E$, dann nennen wir $N$ ein Kernelement von $\mathcal{B}$ und bezeichnen mit $N \in \Re_{p}$. Dabei sei $N \bar{\in}\}$ und für $g=1[N, G]=E$. Dagegen bezeichnen wir mit $N \in Z_{p}$, falls $|N|=p$ und $N \in B$ ist. Ist $\&$ regülär (im Sinne von P. Hall s. Satz (A) in 1) und $|G| \leqq p^{\alpha}$, bilden $G$ und $E$ eine charakteristische Untergruppe von $(B)$ die wir mit $\Omega_{\alpha}(\&)$ schreiben. In 3 sind alle betrachteten Gruppen $p$-Gruppe von der Klasse 2 für $p>2$ und wir schreiben $|A|=p^{a},|B|=p^{b}, \ldots$ für jedes $A, B, \ldots \in \mathcal{B}$, außer wenn wir besonders angeben werden.

Received June 4, 1965. 


\section{Reguläre $p$-Gruppe}

Wir benutzen oft die Sätze von P. Hall, die die Struktur der regulären $p$-Gruppe ans Licht kommen lassen. Daher wollen wir diese Sätze hervorheben. Als eine Charakterisierung der regulären $p$-Gruppe gilt der

SATz (A). B ist genau dann regulär, wenn für jedes $A$ und $B(A B)^{p}=$ $A^{p} B^{p} C^{p}$ mit $C \in\{A, B\}^{\prime}$ gilt.

Hiernach nehmen wir $\$$ reguläre $p$-Gruppe an. Das Ähnliche wie der Basissatz der abelschen Gruppe ist der

SATZ (B). In \& gibt es das eingeordnete Elementsystem $A_{1}, A_{2}, \ldots, A_{t}$ derart, daß jedes $G \in B$ in Form $G=A_{1}^{x_{1}} A_{2}^{x_{2}} \cdots A_{t}^{x_{t}}$ eineindeutig ausgedrückt wird.

Wir nennen dieses Elementsystem eine Basis von (\$. Weiter folgt der

Satz (C). Die Basis von $\mathbb{B}$ kann in folgender Art aufgenommen werden.

1) $\left|A_{1}\right| \geqq\left|A_{2}\right| \geqq \cdots \geqq\left|A_{t}\right|$

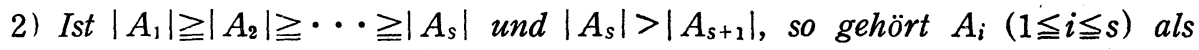
Vertreter genau einem Element der Basis von $\mathbb{B} / \Omega_{\mu-1}(\mathbb{B})$, wobei $|G|=p^{\mu}$ für jedes $G \in \mathbb{B}$ gilt.

Aus diesen Sätzen erhalten wir den als Permutationssatz geltenden

Satz (D). Das Elementsystem $A_{\tau(1)}, A_{\tau(2)}, \ldots, A_{\tau(t)}$ ist auch eine Basis von (S), wobei $\tau$ eine Permutation von $1,2, \ldots, t$ bezeichnet.

\section{Hilfssätze}

Es gilt der folgende

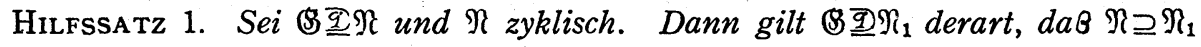
und $\left|\Re_{1}\right|=p$ ist.

Beweis. Wir haben nur $\{G\}_{\vee} \Re_{1}$ für jedes $G \in \mathbb{B}$ zu zeigen. Sei $\mathfrak{R}=\{N\}$ und $\Re_{1}=\left\{N^{p^{n-1}}\right\}$ mit $|N|=p^{n}$. Für beliebiges $G$ von $\mathbb{B}$ gilt $\{G\}_{\mathrm{V}} \Re$. Falls $\{G\} \cap \Re_{F} E$ ist, so folgt $\{G\} \cap \Re \supseteq \Re_{1}$, daher $\{G\rangle \vee \Re_{1}$. Falls $\{G\} \cap \Re=E$ ist, so betrachten wir ein $\mathfrak{g} \subseteq\{G\} \Re$ derart, daB $[\mathfrak{g}:\{G\}]=p$ ist. $\quad$ Da $|\mathfrak{g} \cap \mathbb{M}|=p$ ist, gilt $\mathfrak{g} \cap \mathfrak{\Re}=\left\{N^{p^{n-1}}\right\}$ und $\mathfrak{\mathscr { V }}=\left\{N^{p^{n-1}}\right\}\{G\}=\mathfrak{\Re}_{1}\{G\}$, daher $\{G\}_{\vee} \Re_{1}$.

Wie wir aus dem Beweis ersehen können, gilt Hilfssatz 1 für allgemeine endliche $p$-Gruppe.

Nun kommen wir zum Beweis des grundlegenden Hilfssatzes, der recht 
komplizierte Rechnung erfordert.

Hilfssatz 2. Sei $\mathbb{B}=\{A, B\}$ derart, daB $|A|=|B|=|C|=p^{n}$ mit $[A, B]=$ $C$ ist, und $\mathbb{Q}$ ?. Dann gilt

1) Ist $A^{x} B^{y} \in 3$, so ist $A^{x}=B^{y}=E$.

2) $\mathbb{B}^{\prime}=3$.

3) $\mathfrak{R} \cap 3 \neq(5$.

Beweis. 1) Es ist $B^{\prime} \subseteq 3$, daher $B^{\prime}=\{C\}$. Für jedes $G \in \mathbb{S}$ haben wir einen Ausdruck $G=A^{x} B^{y} C^{z}$. Sei $G \in 3$, dann ist $\left[A, A^{x} B^{y} C^{z}\right]=\left[B, A^{x} B^{y} C^{z}\right]=E$. Also haben wir $[A, B]^{y}=C^{y}=\left[B, A^{x}\right]=C^{-x}=E$, daher $x \equiv y \equiv 0\left(p^{n}\right)$. Folglich gilt $A^{x}=B^{y}=E$.

2) Es ist $\left(\mathcal{S}^{\prime} \subseteq 3\right.$. Sei $G=A^{x} B^{y} C^{z} \in 3$, dann ergibt sich aus 1) $A^{x}=B^{y}=E$ also $G=C^{z} \in \mathbb{B}^{\prime}$. Dies liefert uns $\mathbb{B}^{\prime} \supseteq 3$, daher $\mathbb{B}^{\prime}=3$.

3) Wir zeigen die Eindeutigkeit des Ausdrucks $G=A^{x} B^{y} C^{z}$. Sei $A^{x_{1}} B^{y_{2}} C^{z_{1}}$ $=A^{x_{2}} B^{y_{2}} C^{z_{2}}$, dann erhalten wir $A^{x_{1}-x_{2}} B^{y_{1}-y_{2}} C^{z_{1}-z_{2}}=E$, daher $A^{x_{1}-x_{2}}=B^{y_{1}-y_{2}}=E$ nach 1). Daraus folgt $A^{x_{1}}=A^{x_{2}}$ und $B^{y_{1}}=B^{y_{2}}$, also $C^{z_{1}}=C^{z_{2}}$. Hiernach führen wir unter der Annahme $\mathfrak{N} \cap 3=$ E einen Widerspruch. Da $\left(\mathcal{S} / \mathbb{S}^{\prime} \supseteq \mathfrak{N} \mathbb{S}^{\prime} / \mathbb{S}^{\prime} \simeq \mathfrak{N} / \mathfrak{N} \cap \mathbb{3}\right.$ ist, ist $\mathfrak{R}$ abelsch mit den höchstens zwei bestehenden Erzeugenden. Sei $\Re=\{N\}$ mit $|N|=p^{m}$. Aus Hilfssatz 1 folgt $\mathbb{Q} \underline{Q}\left\{N^{p^{m-1}}\right\}$, daher $\left\{N^{p^{m_{-}-1}}\right\} \mathrm{v}\{A\}$ und $\left\{N^{p^{m-1}}\right\}_{\mathrm{v}}\{B\}$. Das ergibt $\left[N^{p^{m-1}}, A\right] \in\left\{N^{p^{m-1}}, A\right\}^{\prime} \subseteq\{A\}$. Aus 1) haben wir $\{A\} \cap 3=\{A\} \cap \mathbb{S}^{\prime}=\mathbb{E}$ und $\left[N^{p^{m-1}}, A\right]=E$. Daraus folgt auch $\left[N^{p^{m-1}}, B\right]$ $=E$, also $N^{p^{m-1}} \in 3$, was $\mathfrak{R} \cap 3 \neq \mathbb{E}$ führt. Wir nehmen also $\mathfrak{N}=\left\{N_{1}\right\} \times\left\{N_{2}\right\}$ mit $N_{1} \neq E$ und $N_{2} \neq E$ an. Im folgenden sei $i=1,2$ oder $i=1, j=2$ und $i=2$, $j=1$, falls nichts besonders ausgesagt wird. Es gebe die Ausdrücke $N_{i}=$ $A^{x_{i} p^{\alpha_{i}}} B^{y_{i} p^{\beta_{i}}} C^{z_{i} p^{\gamma_{i}}}$, wo $x_{i}, y_{i}, z_{i}$ nicht negative ganzrationale Zahlen modulus $p^{n}$ aufnehmen und $n>\alpha_{i} \geqq 0, n>\beta_{i} \geqq 0, n>\gamma_{i} \geqq 0$ gelten. Dann ist nun $N_{i}^{p^{\delta_{i}}}=$

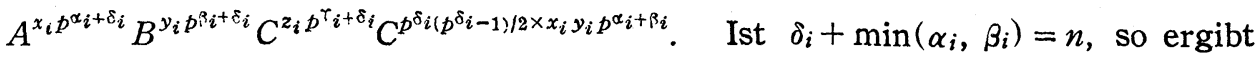
$\operatorname{sich} N_{i}^{\phi_{i}}=C^{z_{i} p^{\gamma_{i}+\delta_{i}}}$. Sei $\gamma_{i}<\min \left(\alpha_{i}, \beta_{i}\right) \quad(i=1$ oder 2$)$, dann ist $C^{z_{i} p^{\gamma_{i}+\delta_{i}}} \neq E$ ( $i=1$ oder 2 ), daher $\Re \cap B \neq \mathbb{E}$ und wir schließen $\gamma_{i} \geqq \min \left(\alpha_{i}, \beta_{i}\right)$.

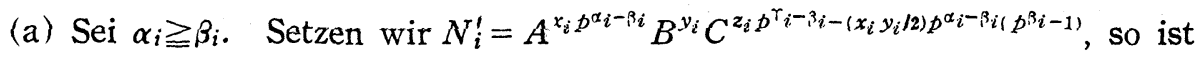

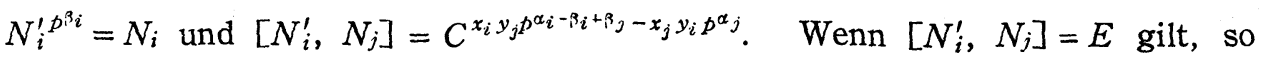
bekommen wir gleich $x_{i} y_{j} p^{\alpha_{i}-\beta_{i}+\beta_{j}}=x_{j} y_{i} p^{\alpha_{j}}\left(p^{n}\right)$. Ist $\beta_{j} \geqq \beta_{i}$, so ergibt sich

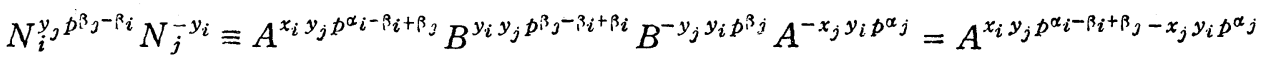
$=E$ modulus 3 , entgegen $\Re \cap 3=\sqrt{5}$ oder $\left\{N_{1}\right\} \cap\left\{N_{2}\right\}=\mathbb{F}$. In diesem Fall sei $i=1$, 
$j=2$ oder $i=2, j=1$.

(b) Sei $\alpha_{i} \leqq \beta_{i}$. 'Wir erwähnen diesen Teil am Schluß.

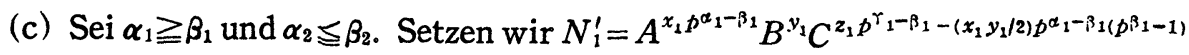

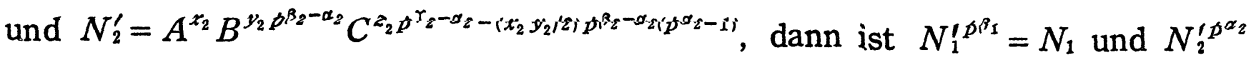
$=N_{2}$. Weiter ist

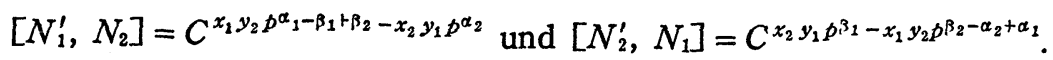

Für $\left[N_{i}^{\prime}, N_{j}\right]=E$ ist $x_{1} y_{2} p^{\alpha_{1}-\beta_{1}+\beta_{2}} \equiv x_{2} y_{1} p^{\alpha_{2}}, x_{2} \cdot y_{1} p^{\beta_{1}} \equiv x_{1} y_{2} p^{\beta_{2}-\alpha_{2}+\alpha_{1}}\left(p^{n}\right)$. Für $\beta_{2} \geqq \beta_{1}$ gilt $N_{1}^{y_{2} p^{\beta_{2}-\beta_{1}}} N_{2}^{-y_{1}}=A^{x_{1} y_{2} p^{\alpha_{1}-\beta_{1}+\beta_{2}}} B^{y_{1} y_{2} p^{\beta_{2}-\beta_{1}+\beta_{1}}} B^{-y_{1} y_{2} p^{\beta_{2}}} A^{-x_{2} y_{1} p^{\alpha_{2}}} \equiv E$ modulus 3 und $\beta_{1} \geqq \beta_{2}, \alpha_{1} \geqq \beta_{1} \geqq \beta_{2} \geqq \alpha_{2}$ ergibt $\alpha_{1} \geqq \alpha_{2}$. Diesmal betrachten wir $N_{1}^{-x_{2}} N_{2}^{x_{1} p^{\alpha_{1}-\alpha_{2}}}$ und haben den gleichen Widerspruch.

(d) Sei $\alpha_{1} \leqq \beta_{1}$ und $\alpha_{2} \geqq \beta_{2}$. Wenn wir $A$ mit $B$ und $\alpha_{i}$ mit $\beta_{i}$ vertauschen, so können wir (b), (d) auf je (a), (c) zurückführen.

Es bleibt noch in allen Teilen den Fall $\left[N_{i}^{\prime}, N_{j}\right] \neq E(i=1, j=2$ oder $i=2$, $j=1)$. Es gilt ersichtlich $\left[N_{i}^{\prime}, N_{j}\right] \in \mathbb{S}^{\prime} \cap\left\{N_{i}^{\prime}\right\} \Re$, daher $\left[N_{i}^{\prime}, N_{j}\right]=C^{\alpha_{i j}}=$ $N_{i}^{\prime}{ }^{y_{i}} N_{1}^{x_{i j}(1)} N_{2}^{x_{i j}(2)}$, da $\left\{N_{i}^{\prime}\right\} \mathfrak{N}$ eine $p$-Gruppe ist. Weiter ist $N_{i}^{\prime y_{i}} \bar{\in} \mathfrak{\Re}$. Sonst wäre $\Re \cap \mathbb{S}^{\prime}=\Re \cap 3 \neq \mathbb{F}$. Wir betrachten $N_{i}^{\prime y_{i}}$. Dann ist $\left(N_{i}^{\prime y_{i}}\right)^{p^{s_{i}-1}}=C^{\alpha_{i j} p^{s_{i}-1}}\left(N_{1}^{x_{i j}(1)}\right.$ $\left.N_{2}^{x_{i j}^{(2)}}\right)^{-p^{s_{i}-1}} \in \Re$ für $\left|N_{i}^{\prime y_{i}}\right|=p^{s_{i}}$ und $\left|N_{i}^{\prime y_{i}}\right|>\left|N_{i}^{-x_{i j}(i)}\right|$, da $\left\{N_{i}^{\prime}\right\} \supseteq\left\{N_{i}^{\prime y_{i}}\right\} \supseteq\left\{N_{i}\right\}$ und $N_{i}^{\prime y_{i}} \in \mathfrak{N}$ ist. Das ergibt $N_{1}^{\prime y_{1} p^{s_{1}-1}}=C^{\alpha_{12} p^{s_{1}-1}} N_{2}^{-x_{22}(2) p^{s_{1}-1}}$ oder $N_{2}^{y_{2} p^{s_{2}-1}}=C^{\alpha_{21} p^{s_{2}-1}}$ $N_{1}^{-x_{21}(1) p^{s_{2}-1}}$. Berücksichtigen wir $E \neq N_{i}^{\prime} y_{i p^{s_{i}-1}} \in \mathfrak{N}$, so gilt $\mathfrak{R} \cap \mathbb{Z} \neq \mathbb{E}$ oder $\left\{N_{1}\right\} \cap\left\{N_{2}\right\} \neq \mathbb{E}$, entgegen der Annahme.

\section{Weiter geiten die folgende}

HILFSSATZ 3. Sei $\mathbb{B} \mathfrak{N}$ und $\mathbb{B}=\mathfrak{g} \mathfrak{N}$ mit $\mathfrak{S} \cap \mathfrak{N}=\mathbb{B} \cap \mathfrak{N}=\mathbb{E}$. Weiter sei $=\{A, B\}$ derart, daB $|A|=|B|=|C|=p^{n}$ mit $C=[A, B]$ ist. Dann gilt $\mathbb{B}=$

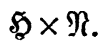

Beweis. Durch Induktion von $|ß|$. Wenn $|\mathfrak{N}|=p$ und daher $\Re=\{N\}$ ist, so gilt $\{N\}_{\vee}\{A\}$ und $\{N\}_{\vee}\{B\}$. Daraus folgt $[N, A]=E$ oder $[N, A]=A^{x p^{a-1}}$ und $[N, B]=E$ oder $[N, B]=B^{y p^{a-1}}$. Aus 1) und 2) in Hilfssatz 2 haben wir $\{A\} \cap \mathbb{S}^{\prime}=\{B\} \cap \mathbb{S}^{\prime}=\mathfrak{E}$, daher $[N, A]=[N, B]=E$ : denn $\mathbb{B}^{\prime} \cap \mathscr{S}$ ist im Zentrum von $\mathfrak{S}$ enthalten. Das ergibt sofort $\mathbb{B}=\mathfrak{S} \times \mathfrak{N}$. Also nehmen wir $|\mathfrak{N}|>p$ an. Wir betrachten $\mathfrak{M}=\mathfrak{S}_{1}$ mit $\mathfrak{N} \supseteq \mathfrak{N}_{1}$ und $\left|\mathfrak{N}_{1}\right|=p . \quad$ Da $\left|\mathfrak{N}_{1}\right|=p$ ist, ergibt sich $\mathfrak{R}=\mathfrak{g} \times \mathfrak{N}_{1}$. Daher ist $\mathfrak{R}_{1} \subseteq \mathcal{Z}$, weil $\mathfrak{R}$ abelsch ist. Es gilt eben durch Induktions-

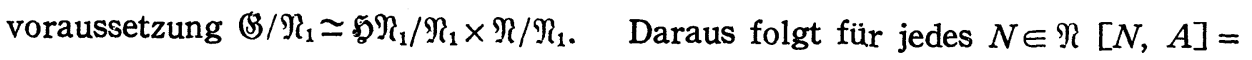
$V^{\alpha}$ und $[N, B]=V^{\beta}$ mit $\mathfrak{R}_{1}=\{V\}$, daher $\mathbb{B}=\mathfrak{S} \times \mathfrak{N}$, da $\mathcal{B}^{\prime} \cap \Re=\mathbb{E}$ ist. 
Hilfssatz 4. Sei $\{A\} \cap\{B\}=\mathbb{E}$ und $|N|=p$ für $A, B, N \in \$$ derart, daB $\{A\}_{\mathrm{v}}\{N\},\{B\}_{\mathrm{v}}\{N\},\{A B\}_{\mathrm{v}}\{N\}$ gelten. Dann ist die Behauptung:

(1) Ist $|A|=|B|$, so ist $[N, A]=[N, B]=E \operatorname{oder}\left[N^{x}, A\right]=A^{p^{a-1}}$ und $\left[N^{x}, B\right]$ $=B^{p^{a-1}}$.

(2) Ist $|A|>|B|$, so ist $[N, A]=[N, B]=E \operatorname{oder}\left[N^{x}, A\right]=A^{p^{a-1}}$ und $\left[N^{x}, B\right]$ $=E$.

wobei $x \neq 0(p)$ ist und $[N, G]=G^{p^{g-1}}$ für $g=1[N, G]=E$ bedeuten soll.

Beweis. Wir betrachten $\mathfrak{U}=\{A\}\{N\}$ und $\mathfrak{B}=\{B\}\{N\}$. Ersichtlich gilt $[N, A]=E$ für $\{N\} \cap\{A\} \neq$ ङ. Dagegen ist $[N, A]=A^{\alpha p^{\alpha-1}}$ für $\{N\} \cap\{A\}=\mathfrak{F}$ : denn es ist $|\{A, N\}|:|A|=p$, also $\{A, N\} D\{A\}$. Gleiches gilt für $[N, B]$.

Von nun ab wird $\alpha \beta \gamma \neq 0(p)$ angenommen.

(1) Nun sei $[N, A]=A^{\alpha p^{\alpha-1}}$ für $a>1$ und $[N, B]=E$. Dann gilt $[N, A B]$ $=[N, A][N, B]=A^{\alpha p^{a-1}}=(A B)^{\Upsilon p^{a-1}}=A^{\Upsilon p^{\alpha-1}} B^{\alpha p^{a-1}}\left[A^{\delta}, B\right]^{p^{\alpha-1}}=A^{\gamma p^{a-1}} B^{\tau p^{a-1}}, \mathrm{da}$ $A^{\delta p^{a-1}} \in\left(B^{\prime} \subseteq 3\right.$ ist, entgegen $\{A\} \cap\{B\}=\mathbb{F}$ oder $a>1$. Ebenso $[N, A]=E$ und $[N, B]=B^{\beta p^{\alpha-1}}$ auch nicht gelten. Nächstens gelte $[N, A]=A^{\alpha p^{\alpha-1}}$ und $[N, B]$ $=B^{\beta p^{\alpha-1}}$. Dann muB $[N, A B]=A^{\alpha p^{a-1}} B^{\beta p^{\alpha-1}}=(A B)^{\gamma p^{\alpha-1}}=A^{\gamma p^{\alpha-1}} B^{\gamma p^{\alpha-1}}$ sein, was besagt $\alpha \equiv \beta \equiv \gamma(p)$, also $\left[N^{x}, A\right]=A^{p^{a-1}}$ und $\left[N^{x}, B\right]=B^{p^{\alpha-1}}$ für $\alpha x \equiv 1(p)$.

(2) Diesmal sei $[N, A]=E$ und $[N, B]=B^{\beta p^{b-1}}$ für $b>1$. Dann haben wir $[N, A B]=[N, A][N, B]=B^{\beta p^{b-1}}=(A B)^{\Upsilon p^{a-1}}=A^{\Upsilon p^{a-1}} B^{\Upsilon p^{a-1}}=A^{\Upsilon p^{a-1}}$, da $b<a$, daher $B^{\beta p^{\alpha-1}}=E$ ist. Hieraus folgt $A^{\Upsilon p^{\alpha-1}}=B^{\beta p^{b-1}}$, entgegen $\{A\} \cap\{B\}=\&$.

Hilfssatz 5. Seien $A_{1}, A_{2}, \ldots, A_{t}$ eine Basis von $\left(3 \mathrm{mit}\right.$ der Ordnung $\left|A_{i}\right|$ $=p^{\mu_{i}}=p^{\mu}$ für $1 \leqq i \leqq s$, bzw. $\quad\left|A_{j}\right|=\left|A_{s+1}\right|=p^{\mu_{2}}<p^{\mu}$ für $j>s$ (s. Satz (C) in 2). Für ein $N \in \mathbb{B}$ mit $|N|=p$ sind die zwei folgenden Aussagen gleichwertig.

(1) $N \in \Re_{p}$

(2) $\left[N, A_{i}\right]=A_{i}^{p^{a-1}}(1 \leqq i \leqq s)\left[N, A_{j}\right]=E(j \geqq s+1)$.

Beweis. Aus (1) folgt (2). Da $N \bar{\Xi} 3$ ist, gibt es wenigstens ein $A_{i}(1 \leqq i \leqq t)$ mit $\left[N, A_{i}\right] \neq E$. Aus Hilfssatz 4 folgt sofort (2).

Aus (2) folgt (1). Da $A_{1}, A_{2}, \ldots, A_{t}$ die Basis von $\&$ ist, haben wir für jedes $G \in \dot{B}$ einen Ausdruck $G=A_{1}^{x_{1}} A_{2}^{x_{2}} \cdots A_{t}^{x_{t}}$ von $G$. Aus $\left\{A_{i}\right\} \cap 3 \supseteq\left\{A_{i}\right\} \cap \notin$ $\neq\left(5\right.$ und $\left|A_{j}\right| \leqq p^{a-1}$ folgt $\left[A_{i_{1}}, A_{i_{2}}\right]^{p^{a-1}}=E\left(1 \leqq i_{1}, i_{2} \leqq t\right)$, daher $G^{p^{a-1}}=A_{1}^{x_{1} p^{a-1}}$ $A_{2}^{x_{2} p^{a-1}} \cdots A_{t}^{x_{t} p^{a-1}}=A_{1}^{x_{1} p^{a-1}} A_{2}^{x_{2} p^{a-1}} \cdots A_{s}^{x_{s} p^{a-1}}:$ denn es ist $A_{j} \in \Omega_{a-1}(\dot{\xi})$. Andererseits haben wir $[N, G]=A_{1}^{x_{1} p^{\alpha-1}} A_{2}^{x_{2} p^{a-1}} \cdots A_{s}^{x_{s} p^{\alpha-1}}$ also $[N, G]=G^{p^{\alpha-1}}=G^{p^{\mu-1}}$ 
Das besagt $[N, G]=G^{p^{\mu-1}}$ für $|G|=p^{\mu}$ und $[N, G]=E$ für $|G|<p^{\mu}$, was $N \in \Omega_{p}$ ergibt.

Hilfssatz 6. Sei $\mathbb{Q} \mathbb{M}$ und $|\mathfrak{R}|=p$. Dann gibt es ein $N \in \Re$ derart, daß $N \in 3_{p}$ oder $N \in \Re_{p}$ ist.

Beweis. Sei $A_{1}, A_{2}, \ldots, A_{t}$ eine Basis von $\dot{B}$. Wegen $\mathbb{Q} \mathfrak{M}$ ist $\mathfrak{N v}\left\{A_{i}\right\}$ $(1 \leqq i \leqq t)$, daher nach Hilfssatz $4\left[N, A_{i}\right]=E(1 \leqq i \leqq t)$ oder $\left[N, A_{i}\right]=A_{i}^{p^{u-1}}$ $(1 \leqq i \leqq s)$ und $\left[N, A_{j}\right]=E(s+1 \leqq j \leqq t)$, was $N \in 3_{p}$ oder nach Hilfssatz 5 $N \in \Re_{p}$ ergibt.

Hilfssatz 7. Sei $\mathbb{Q} \nsubseteq \mathfrak{N}$ und $\Re \cap 3=\mathbb{E}$. Dann gilt $\{G\}_{\mathrm{v}}\{N\}$ für jedes $G \in \mathbb{B}$ mit $\{G\} \cap 3 \neq \xi$ und $N \in \mathfrak{R}$ mit $|N|=p$.

Beweis. Ist $[N, G]=C \neq E$, so ist wegen $C \in\{G\} \Re C=G^{\alpha} N_{1}$ mit $N_{1} \in \Re$. Aus $\{G\} \cap \Re \cap Z=\&$ folgt $\left(G^{\alpha}\right)^{p}=\left(C N_{1}^{-1}\right)^{p}=N_{1}^{-p}=E$. Sonst wäre $\{G\} \cap \Re \cap ß$ $\neq$ \&. Hiernach haben wir $G^{\alpha} \in 3$, daher $N_{1}=C G^{-\alpha} \in 3$, was wegen $\Re \cap 3=\mathbb{E}$ $N_{1}=E$ führt. Dies besagt aber $C=G^{\alpha}$ und $\{G\}_{v}\{N\}$.

Da wir diese Hilfssätze bewiesen haben, so untersuchen wir den

Hauptsatz. Sei $\mathbb{(} \underline{\mathbb{Q}} \Re$. Dann gibt es in $\Re$ ein $N$ mit $N \in 3_{p}$ oder $N \in \Omega_{p}$.

Beweis. Sei $A, B \in \dot{B}$. Dann ist $(A B)^{p}=A^{p} B^{p}[A, B]^{p(p-1) / 2}=A^{p} B^{p}([A$, $\left.B]^{\dot{p}-1 / 2}\right)^{p}$, da $p>2$ ist. Nach Satz (A) ist $\&$ regulär. Daher hat eine Basis $A_{1}, A_{2}, \ldots, A_{t}$ wie in Hilfssatz 5. Wir führen den Beweis durch Induktion von $|\$|$. Wegen der Auswahl von $\operatorname{der}$ Basis $A_{1}, A_{2}, \ldots, A_{t}$ können wir $A_{1}$, $A_{2}, \ldots, A_{s}$ als Basisvertreter $\operatorname{der}\left(\mathbb{S} / \mathbb{S}^{\prime} \Omega_{\mu-1}(\mathbb{B})\right.$ und $\mathbb{S}^{\prime} \Omega_{\mu-1}(\mathbb{B}) / \Omega_{\mu-1}(\mathbb{B})$ aufnehmen und nach Satz $(D) A_{1} \in \mathbb{S}^{\prime} \Omega_{\mu-1}(\mathbb{B})$ annehmen. Daher gibt es in $\mathbb{B}$ ein maximales $\mathfrak{M}$, das $A_{1}^{p}, A_{2}, \ldots, A_{t}$ enthält. Wir betrachten den Ausdruck $M=A_{1}^{x_{1}} A_{2}^{x_{2}} \cdots A_{t}^{x_{t}}$ für jedes $M \in \mathfrak{M}$. Ersichtlich gilt $A_{1}^{x_{1}} \in \mathfrak{M}$, da $A_{2}, A_{3}, \ldots$, $A_{t} \in \mathbb{M}$ ist, daher $x_{1} \equiv 0(p)$. Dies spricht aus, daB $A_{1}^{p}, A_{2}, \ldots, A_{t}$ eine Basis von $\mathfrak{M}$ ist. Nach Satz (D) haben wir, daB $A_{2}, A_{3}, \ldots, A_{s}, A_{1}^{p}, \ldots, A_{t}$ auch eine Basis von $\mathfrak{M}$ ist: demnach genügt $\mathfrak{M}$ Induktionsvoraussetzung und wir können aus dem Hilfssatz $6 \mathfrak{M} \cap \mathfrak{N} \mathbb{E}$ annehmen. Daher enthält $\mathfrak{M} \cap \mathfrak{R}$ erst recht $\Re$ ein Zentrumselement oder Kernelement $N$ von $\mathfrak{M}$. Wir nennen ein $N \in 3_{p}$ kurz ein Zentrumselement von $\mathbb{B}$.

(1) Sei $\left\{A_{1}\right\} \cap \mathbb{Z}=\mathbb{E}$, daher $\left|A_{1}\right|=\left|A_{2}\right|=\left|\left[A_{1}, A_{2}\right]\right|$. Wir betrachten $\mathfrak{V}=$ $\left\{A_{1}, A_{2}\right\}, \mathfrak{B}=\mathfrak{U} \mathfrak{N}, \mathfrak{N}_{1}=\mathfrak{A} \cap \mathfrak{N}, \quad$ Ist $\mathfrak{R}_{1} \neq \mathfrak{F}$, so haben wir $\{A\rangle \vee \mathfrak{N}_{1}$ für jedes $A$ 
$\in \mathfrak{A}$, da $\{A\} \mathfrak{R} \cap \mathfrak{U}=\{A\} \mathfrak{N}_{1}$ ist. $\quad$ Es gilt nun $3 \cap \mathfrak{⿰} \supseteq \mathbb{S}^{\prime} \cap \mathfrak{⿰} \supseteq \mathfrak{U}^{\prime} \cap \mathfrak{R}_{1}$ und nach Hilfssatz $2 \mathfrak{I}^{\prime} \cap \mathfrak{R}_{1} \neq \mathfrak{F}$ : demnach $3 \cap \mathfrak{R} \neq \mathfrak{F}$. Von nun ab haben wir nur den Fall $\mathfrak{A} \cap \mathfrak{N}=\mathfrak{E} z \mathrm{u}$ behandeln. Das ergibt aber nach Hilfssatz $3 \mathfrak{B}=\mathfrak{A} \times \mathfrak{N}$, da $\mathfrak{B}^{\prime} \cap \mathfrak{N} \subseteq \mathfrak{B}^{\prime} \cap \mathfrak{R} \subseteq 3 \cap \mathfrak{R}$ gilt und wir $3 \cap \mathfrak{R}=\mathfrak{F}$ annehmen können. Es werde angenommen, daB $N$ ein Zentrumselement von $\mathfrak{M}$ ist. $\quad \mathrm{Da}\left[N, A_{1}\right]=E$ ist, ist $N \in 3 p$. Falls $N$ dagegen ein Kernelement von $\mathfrak{M}$ ist, so ergibt sich nach Hilfssatz $5\left[N, A_{2}\right]=A_{2}^{p^{\alpha-1}}$, entgegen $\left[N, A_{2}\right]=E$.

(2) Sei $\left\{A_{1}\right\} \cap 3 \neq\left(\mathbb{E}\right.$ und $\left\{A_{i_{1}}\right\} \cap B=\mathbb{E}$ für ein $i_{1}$ mit $\left(1<i_{1} \leq s\right)$. Nach der Auswahl von der Basis können wir $A_{1} A_{i_{1}}, A_{2}, \ldots, A_{t}$ auch als Basis von $\&$ aufnehmen. Da $\left(A_{1} A_{i_{1}}\right)^{p^{a-1}}=A_{1}^{p^{\alpha-1}} A_{i_{1}}^{p^{\alpha-1}}\left[A_{1}, A_{i_{1}}\right]^{\left(p^{a-1}\right)}$ ist, haben wir $\left\{A_{1} A_{i_{1}}\right\} \cap 3$ =․ Daher können wir (2) auf (1) zurückführen.

(3) Sei $\left\{A_{i}\right\} \cap 3 \neq \mathbb{E}(1 \leqq i \leqq s$ und $s=1)$. Es ist $\{N\} \mathrm{v}\left\{A_{i}\right\} \quad(2 \leqq i \leqq t)$ und nach Hilfssatz $7\{N\}_{\vee}\left\{A_{1}\right\}$. Für $1 \leqq i \leqq t$ gilt $\left[N, A_{i}\right]=E$ oder nach Hilfssatz $5\left[N^{x}, A_{1}\right]=A_{1}^{p^{a-1}}$ und $\left[N, A_{i}\right]=E(2 \leqq i \leqq t)$. Hilfssatz 6 liefert $N \in 3_{p}$ oder $N^{x} \in \Omega_{p}$.

(4) Sei $\left\{A_{i}\right\} \cap 3 \neq(1 \leqq i \leqq s$ und $s>1)$. Es ist $\{N\}_{\mathrm{v}}\left\{A_{j}\right\} \quad(s+1 \leqq j \leqq t)$ und nach Hilfssatz $7\{N\}_{\mathrm{v}}\left\{A_{i}\right\}$ und $\{N\}_{\mathrm{v}}\left\{A_{1} A_{i}\right\} \quad(1 \leqq i \leqq s)$. Da $\left|A_{i}\right|>\left|A_{j}\right|(1 \leqq i$ $\leqq s$ und $s+1 \leqq j \leqq t)$ ist, erhalten wir $\left[N, A_{i}\right]=E(1 \leqq i \leqq t)$ oder nach Hilfssatz 5 $\left[N^{x}, A_{i}\right]=A_{i}^{p^{a-1}}$ und $\left[N^{x}, A_{j}\right]=E$. Daher ist $N \in 3_{p}$ oder nach Hilfssatz 7 $N^{x} \in \Re_{p}$.

\section{LiTERATUR}

[1] Hall, M.: The theory of groups. New York, 1959.

[2] Hall, P.: A contribution to the theory of groups of prime power order. Proc. Lond. Math. Soc., (2) 36, 29-95 (1933).

[3] Zassenhaus, H.: Lehrbuch der Gruppentheorie I., 1937.

Mathematisches Institut der Nihon-Universität Koriyama (Japan) 